\title{
Zwischen Empirie und Magie: Die mittelalterliche Frakturbehandlung durch die Laienpraktiker*
}

\author{
Von Volker Zimmermann
}

Die Frakturbehandlung im Mittelalter wird nicht allein durch die einschlägigen Kapitel in den großen Chirurgien eines Bruno von Longoburgo, eines Lanfrank von Mailand, eines Henri de Mondeville oder eines Guy de Chauliac bestimmt ${ }^{1}$, sie spiegelt sich auch in einer Vielzahl von Einzelrezepten und Rezeptblöcken wider, die, in umfängliche Sammelhandschriften eingestreut, tradiert werden. Diese Arzneimittel stellen eine bislang nicht beachtete Ergänzung zu den mechanisch chirurgischen Maßnahmen der Reposition und Fixation dar. Die Edition dieser ausnahmslos landessprachigen Texte steht erst am Anfang. Die folgenden Untersuchungen, die sich auf hier erstmals edierte Textzeugen stützen, wollen das Aufgabenfeld konturieren und erste Markierungspunkte setzen.

Die Sammelhandschriften stammen aus der zweiten Hälfte des Mittelalters. Ihre in der Regel anonymen Kompilatoren haben in enzyklopädischer Form Schriften aus den verschiedenen «artes liberales et mechanicae» zusammengetragen. Die Grundstruktur der Kompendien besteht aus Texten der vierten, fünften und sechsten Eigenkunst, wobei die human-medizinischen Schriften den weitaus größten Raum einnehmen. In diesem Zusammenhang spielen neben den beiden wirkungsmächtigsten mittelalterlichen Arzneibüchern des Bartholomäus und des Ortolfs von Baierland, neben Traktaten über verschiedene Krankheiten, wie z. B. die Pest, vor allem die umfangreichen, anonym abgefaßten Rezeptkollektaneen eine wichtige Rolle $^{2}$. In diesen Rezeptsammlungen werden auch Einzelrezepte tradiert, die Verfahren zur Behandlung von Arm- und Beinbrüchen beschreiben. Wie dies im einzelnen aussah, soll im folgenden an spezifischen Rezepten aus einer Salzburger Handschrift ${ }^{3}$ und einer Handschrift aus dem ehemaligen Benediktinerkloster Farfa in Latium, die heute in Rom aufbewahrt wird ${ }^{4}$, demonstriert werden.

\footnotetext{
* Erweiterte und mit Fußnoten versehene Fassung eines Vortrages, der auf der 166. Jahrestagung der Schweizerischen Naturforschenden Gesellschaft 1986 in Bern unter dem Titel «Die Frakturbehandlung im Mittelalter» gehalten wurde.
} 
Die beiden Quellen tradieren insgesamt 38 Textzeugen ${ }^{5}$. Davon bieten 34 Kataplasmen für die Frakturbehandlung an; drei einen Saft zum Einnehmen und eines enthält ein Verfahren, das sowohl die Herstellung eines Saftes als auch eines Pulvers ermöglicht.

Bei keinem Rezept wird unterschieden, ob es sich um einen offenen oder geschlossenen Bruch handelt. Doch läßt sich aufgrund der jeweils benutzten Heilpflanzen, die - wie unten zu zeigen sein wird - auch zur Wundbehandlung dienten, eine gewisse Differenzierung erkennen. Auch ermöglicht die Quellenlage bislang noch keine abschließende Antwort auf die Frage, ob die Anwendung der Kataplasmen, Säfte und Pulver die mechanischen Mittel der Reposition und Fixation ergänzen oder ob sie eine eigenständige Heilungsmethode darstellen. Im Salzburger Kompendium ist einmal der Hinweis auf die Reposition belegt. «solt du ym daz bein ziehen also daz es glich stee. Dar nach so nym ... bynwelle gepulfert mit warmem waszer ...» $\left(\mathrm{Bl} .204^{\mathrm{va}}\right)$. Bevor also die Fraktur mit einem Kataplasma behandelt wird, soll das Bein so lange gezogen werden, bis es gerade ist.

Auf die Fixation wird dagegen bei keinem der hier untersuchten Textzeugen hingewiesen. Demgegenüber enthalten aber mehrere Kataplasmen Stoffe wie Wachs, Pech, verschiedene Harzarten, Mehl oder Eier, die beim Trocknungsvorgang ohne Zweifel eine Härtung des Kataplasmas bewirken. Einige Rezepte erwähnen darüber hinaus Tücher, die vor, während oder nach dem Auftragen des Kataplasmas um die Bruchstelle gewickelt werden sollen. Dadurch wird die Festigkeit noch erheblich erhöht, so daß eine zusätzliche Fixation als nicht mehr nötig erscheint.

Die Tatsache, daß die mechanischen Mittel mit einer einzigen Ausnahme hier nicht belegt sind, sowie das Verordnen von Härtungssubstanzen und Stützmitteln in mehreren Rezepten lassen wohl den Schluß zu, daß die Bruchrezepte des Spätmittelalters mit großer Wahrscheinlichkeit eine eigenständige Methode der Frakturbehandlung darstellen.

Wie das Rezept hergestellt wird, ist genau festgelegt. In der Regel bestehen die Verfahren aus den folgenden fünf Schritten: Verkleinern, Mischen und Erhitzen der Substanzen, Seihen und Auflegen des Breis. Beim Seihen wird besonderer Wert auf ein neues oder weißes Tuch gelegt. Ein solches Verfahren ist aus heutiger Sicht in mehrfacher Weise bemerkenswert. Sicherlich dient die Maßnahme zunächst dazu, den Sud von den gröberen Bestandteilen zu reinigen, ihn zu verfeinern, und ihn wirkungsvoller zu machen. $\mathrm{Ob}$ sich dahinter auch vielleicht unbewußt die Vorstellung verbirgt, damit könne eine Übertragung von Krankheiten verhindert 
werden, muß bei der gegenwärtigen Quellenlage noch offen bleiben. Die Vermutung liegt nahe, zumal entsprechende Ansätze aus dem Bereich der mittelalterlichen Hygiene bekannt sind ${ }^{6}$.

Die Maßangaben bei der Verwendung der einzelnen Substanzen entsprechen dem mittelalterlichen Standard. Allgemeine Mengenangaben, wie «nim swarcz-wurcz und stoz sie mit honig und ...» stehen neben exakten wie Lot, Unze, Vierling und Pfund.

Außer Angaben, wie lange die jeweilige Behandlung durchgeführt werden muß, tradieren diese Texte zur Frakturbehandlung auch Hinweise über die Heilungsdauer. Sie erstreckt sich auf einen Zeitraum zwischen sieben Tagen und zehn Wochen, wobei zwischen einem Bruch oberhalb und unterhalb des Knies unterschieden wird. Während die Heilung eines Bruches der tibia nur sechs Wochen dauert, beträgt die Heilungsdauer bei einem Oberschenkelbruch zehn Wochen.

In ihrer Grundstruktur bestehen die Frakturrezepte aus mineralischen, tierischen und vor allem pflanzlichen Substanzen. Während die mineralischen Produkte wie kleingeriebenes Bleiwei $\beta^{7}$ oder Spangrün ${ }^{8}$ in erster Linie der Farbgebung dienen, ist die Verwendung der hier belegten Substanzen recht heterogen. Hunde- oder Ochsenschmalz, Hammel- oder Hirschunschlitt verschmelzen die Teilprodukte zu einem Brei, machen ihn streichfähig und fördern darüberhinaus seine Haltbarkeit. Pulverisierte Geierknöchelchen als Mittel der Frakturbehandlung belegt bereits die hochmittelalterliche Drogenmonographie über den Geier ${ }^{9}$. Neu ist dagegen in diesem Zusammenhang die Verwendung von Bibergeil. Pulverisierte Biberhoden dienen in der mittelalterlichen Rezeptliteratur sonst üblicherweise als Aphrodisiakum ${ }^{10}$. Ein signifikantes Beispiel für Simile-Magie ist die Benutzung der Haut eines Hundewelpens. Dahinter verbirgt sich die Vorstellung eines Analogiezaubers. So wie die Haut den jungen Hund einhüllt und schützt, so soll der gebrochene Knochen durch die um ihn gewickelte Hundehaut geschützt und seine Heilung gefördert werden.

Im Mittelpunkt der pflanzlichen Substanzen stehen Heilpflanzen. Ihnen kommt im Hinblick auf den Heilungsprozeß der Knochenfraktur die entscheidende Rolle zu. An fünf typischen Beispielen soll im folgenden ihre Wirkungsweise näher untersucht werden.

$\mathrm{Zu}$ den Heilpflanzen, die in den mittelalterlichen Bruchrezepten wiederholt belegt sind, zählt der Sanikel (Sanicula europaea L.). Seine heilkundliche Wirkung bei Geschwüren, Blutungen, Wunden und Quetschungen kennt die mittelalterliche Arznei seit der «Physica» Hildegards von Bingen ${ }^{11}$. 
Zu den zentralen pflanzlichen Substanzen gehört auch der Beinwell (Symphytum officinale L.), der in den hier untersuchten Rezepten daneben mit den Synonyma Schwarzwurz oder Wallwurz belegt ist. Ihm wird bereits im «Herbarius» des Pseudo-Apuleius, der im 4. Jahrhundert nach Christi angelegt wurde, wundenheilende Kraft zugeschrieben. In die gleiche Pflanzenfamilie gehört auch die Günsel (Ajuga reptans L.), die mit denselben heilkundlichen Eigenschaften ausgestattet ist ${ }^{12}$.

Die Fähigkeit, Wunden zu heilen, besitzt neben dem Wegerich (Plantago major L.), den bereits Dioscurides als blutstillendes Mittel schätzte ${ }^{13}$, auch der Odermennig (Agrimonia eupatoria L.). Seine große Bedeutung für die mittelalterliche Pflanzenkunde zeigt sich vor allem in der Tatsache, daß ihn Walafrid Strabo, der Abt von Reichenau, in seinen «Hortulus» aufnahm, wo er ihn mit Sarcocola - d.i. Fleischleim - bezeichnete ${ }^{14}$.

Kennzeichnend für alle fünf Pflanzen ist also ihre Fähigkeit, Wunden zu heilen. Während ihre Wirkung als Wundkraut im Mittelalter ausschließlich auf empirischer und magischer Grundlage fußt, wird heute aufgrund chemischer und pharmakologischer Untersuchungen davon ausgegangen, daß die Ursache in den antibakteriellen und antiseptischen Gerb- und Bitterstoffen, in den Saponinen und ätherischen Ölen, die im Rhizom und in den Blättern jeder dieser Pflanzen enthalten sind, zu suchen ist ${ }^{15}$. Neu ist jedoch die in der zweiten Hälfte des Mittelalters auftauchende Verwendung bei Heilung von Beinbrüchen.

Die Ursache für diese zusätzliche Tugend liegt wohl in einem Analogieschluß. Plinius der Ältere berichtet in seiner Naturgeschichte, das Kraut «alum», damit ist Beinwell gemeint, habe zur Heilung von Wunden wohl deswegen eine so vorzügliche Kraft, weil diese Pflanze sogar Fleischstücke, denen sie beim Kochen zugesetzt wird, zusammenklebt; «volneribus sanandis tanta praestantia est, ut carnes quoque, dum cocuntur, conglutinet addita» wie die Stelle im Original heißt ${ }^{16}$. Die gleiche Vorstellung entwickelt - wie bereits erwähnt - Walafrid Strabo im Zusammenhang mit dem Odermennig. Sein Terminus Sarcocola wandelt sich dann in den «Observationes» des Wilhelm Fabry von Hilden in «Osteocola - d.i. Knochenleim». ${ }^{17}$ Unterstützt wird diese These nicht zuletzt auch durch die Tatsache, daß sich die Vorstellung, diese Pflanzen könnten auch gebrochene Knochen wieder zusammenleimen, in der Volksmedizin bis in die Neuzeit hinein gehalten hat. Es gilt also festzuhalten: Die verwendeten Heilpflanzen sind im Zusammenhang mit der Wundbehandlung alle bereits bekannt, sie erweitern jedoch im Zusammenhang mit der Frakturbehandlung in den 
Rezepten aus der zweiten Hälfte des Mittelalters ihre heilkundlichen Tugenden.

Neben dem Analogieschluß spielt in diesem Zusammenhang auch die Wechselbeziehung zwischen Funktionalität und Rezeption dieser Texte eine gewichtige Rolle. Zum Rezipientenkreis zählen nämlich in erster Linie die mittelalterlichen Wundärzte und Bader. Sie benötigen für ihre heilkundliche Aufgabe Rezepte, die in der Landessprache abgefaßt, und deren Substanzen in ihrem Umkreis erhältlich waren. Darüberhinaus schufen diese landessprachigen Texte mit ihrem weitgespannten, auf das Niveau des Laien und Praktikers zugeschnittenen Inhalt einen Kreis von Benutzern, die aus einem vielschichtigen gesellschaftlichen Umfeld stammen ${ }^{18}$. Ihnen bedeuten heimatliche Heilpflanzen, die greifbar sind, deren Wirkung sie aus Erfahrung kennen und an die sie letztlich auch glauben, weit mehr als jede gelehrte lateinische Abhandlung der großen mittelalterlichen Chirurgien, die sie ohnehin nicht verstehen. Wie auch hätte man in der täglichen Praxis ein so kompliziertes mechanisches Verfahren wie das der permanenten Gewichtsextension eines Guy de Chauliac ${ }^{19}$ anwenden sollen?

Die hier untersuchten Rezepte zur Frakturbehandlung, die in verschiedenen Rezeptkollektionen spätmittelalterlicher Sammelhandschriften überliefert werden, wenden sich also nicht an die Angehörigen der Schulmedizin, sondern in erster Linie an die breite Schicht mittelalterlicher Laienpraktiker. Die Rezepttexte zeigen darüberhinaus eine gelungene Symbiose aus empirischem Wissen und magischen Heilvorstellungen, deren Tradition teilweise bis auf antike Quellen zurückreicht. Sie lassen ferner erkennen, daß vor allem im Spätmittelalter neben der mechanischen Frakturbehandlung mit großer Wahrscheinlichkeit auch eine eigenständige, nur auf Arzneimitteln beruhende Heilmethode existiert. 


\section{Edition ausgewählter Rezepte}

Der Abdruck der Texte erfolgt buchstabengetreu. Lediglich die Groß- und Kleinschreibung wird geregelt und die Abbreviaturen aufgelöst. Die Interpunktion wird sparsam verwendet; sie soll in erster Linie das Textverständnis erleichtern. Diakritische Zeichen werden bei lautlicher Relevanz übernommen ${ }^{20}$.

Salzburg: Cod. M. III, 3

Bl. $204^{\text {va }}$

Ein ander capittel von den gebrochen beynen

Zv eym gebrochen beyne solt du ym daz bein ziehen also daz es glich stee. Dar nach so nym consolidam maiorem daz ist swartze beynwelle gepulfert mit warmem waszer vnd gusz es vff ein tuch daz czu nehsten vff dem (Bl. 204 ${ }^{\mathrm{vb}}$ ) bein lit. Der tucher sollent vj sin vnd sollent kurtz sin. Wann man daz bein an dem dritten tage vff bint daz man daz bein nirgent rure. Dar nach so bint es an dem fünfften tage so heylet es wol. Du solt mercken uber vj wochen ist daz es daz beyn zu ym und von ym zuhet, so mag er uff krucken wol gen. Ist es aber ober dem knuwe so musze er sich $\mathrm{x}$ wochen huten.

Item vur gebrochen beyne

Stoss rossloup mit wine vnd mit pfeffer vnd daz drincke viii tage so wollet es zu samen.

$205^{\mathrm{va}}$

$\mathrm{Zu}$ dem bein brůche

So nym iii lot wahsses vnd ein lot wiszes wirauchs vnd linoley ein firtel eins pfundes vnd zerlasze das gar wol in einer pfannen vnd zuch dann ein nuwes duch dar doch vnd daz pflaster horet vber bein brůch daz sol man dar vber legen.

$209^{\text {va }}$

Von bein brechen

Dem ein beyne brichet wo es yme an dem libe sy der neme gutsol die merre vnd lege sie in wine oder in waszer vnd derre sie vnd pulfer sie vnd drincke daz vnd isze das pulfer so fellet daz bein wider vnd heilet. 
$230^{\mathrm{va}}$

Eyn beyn broch pflaster

Wer ein gut beinbroch pflaster pflastere machen wil zum ersten bande der sol nemen j libra sanickel samen vnd sol yn derren vnd pulfern vnde sol nemen swartz bonen vnd sol die sieden bisz daz yn die belge abe fallent vnd sol die pulfern vff einen halben vierling vnde sol nemen $\mathrm{j}$ fierling walwortz vnd sol die auch pulfern vnd $\mathrm{j}$ libra baumoley vnd vj eyer tottern vnd sol die wol zertryben vnd iii loit saltz vnd sol daz in ein becken tune vnd $\left(\mathrm{Bl} 230^{\mathrm{vb}}\right)$ es vndereinander kneden vnd sol so vil zertryben als er bedarff vnd legen vff ein tuch.

$230^{\mathrm{vb}}$

Eyn gute salbe zu einem beinbroch

der sol nemen hundes smalcz von einem bracken $\mathrm{j}$ libra vnd zerlass daz vff einer glut vnd sol daz gieszen vff $\mathrm{j}$ masz wins vnd lasz es stane acht tage vnd dann nym $\mathrm{j}$ libra baumoley $\mathrm{j}$ firling lertschen vnd $\mathrm{j}$ feiszt bybergeyl vnd daz vnder einander tryben.

$256^{\mathrm{ra}}$

Item wil man alle gebrochen beine heylen

So neme linen oley bottern vnd viernen smer vnd rindes marg vnd smeltze viere zu hauff in einer pfannen vnd dan syhen durch ein duch vnd sol nemen wegerich vnde nachtschetten odermenie vnd ybsch wortzelen schelen vnd sol sie dan wol stoszen vnd sol es $\left(256^{\mathrm{rb}}\right)$ alles zu hauff tun vnd dan sihen dorch ein tuch alles mit einander vnd diese salbe ist gut vor geswulste vor schosz die zu den gebrochen beinen slecht.

$396^{\mathrm{rb}}$

Dem ein bein brichet es sy wie es wolle

Der neme eines hundes welpffen der eines dages alt ist vnd sol ym sin hut abe ziehen vnn sol sin hern dar jnne legen vnd sol daz legen uber daz gebrochen beine so heylet es gantz wider.

Oder nym eines ebers speck vnd binde daz vber daz gebrochen beine.

Oder nym krebse vnd stosze die mit eselin milch vnd binde daz da dir we sy. Oder nym die bletter oder rinden von eym eschenbaum vnd siede die mit 
guttem win vnn binde daz off die gebrochen beine alzo warm so wehsset es gantz wyder dustu die artzenie dicke.

Oder nym eines gyren bein vnd pulffer die vnd sewe daz in die wunde so heylt sie reine.

Rom: Cod. 200 aus Farfa

Bl. $98^{\mathrm{r}}$

Vor ver wunte vnd gebrochen beine

Nym weirach weise mirre weisen harczt new wachs vnd seut daz czu samen vnd tu ein schones tuch dorin daz ez wol durch gee vnd loz ez denn erkalden vnde smire denn daz plaster mit aldem smere vnd lege ez warm dorauf da tu allewege wenn du ez doroff legen wilt so werme ez vor so wirt ez weich vnd los das bayn nicht darinne.

$98^{v}$

fur bein broche vnd abgebrochen sint

Nim swarcz wurcz vnd stoz sie mit honig vnn mit pheffer vnd mit wein vnd trinck di bru viiij mol yn dem tage vnd salbe die bein broche mit sanickel saft es heilet wol an enander daz wisse verwore.

99 r

Vor die bein broche

Nym krebs vnd stos sye wol vnd drucke ez durch eyn tuch vnd tu ein eie dor vnder vnd mache doraus ein phlaster vnd binde es uber daz bein so heilet es.

$99^{\mathrm{v}}$

Vor die alden bein brochen

Nym ein lot gestosen segelbawn vnd iij quinten spongrun vnd iij quinten swefbel vnd $\mathrm{j}$ lot lorber stos daz alles yn einem morser gar wol clein vnd nym denn borgel speck vnd czu loz den yn einem phenlein vnn dringe dy griefen wol aus mit einem loffel vnd nym denn dz selbe smalcz vnd temper des obengeschriben puluers do mit daz es wider czu dicke noch czu dunne werde so hostu eyne gute salbe. 


\section{Anmerkungen}

1 Vgl. Volker Zimmermann, Die mittelalterliche Frakturbehandlung im Werk von Lanfrank und Guy de Chauliac. Würzburger medizinhistorische Mitteilungen 6 (1988), S. 5-18.

2 Vgl. ders., Rezeption und Rolle der Heilkunde in landessprachigen handschriftlichen Kompendien des Spätmittelalters (Ars medica. IV. Abt. Landessprachige und mittelalterliche Medizin, Bd.2). Stuttgart 1986, S.1-3.

3 Zur Salzburger Handschrift vgl. Siegfried Sudhof, Ein Salzburger Sammelcodex mittelalterlicher Medizin und Naturwissenschaft. Med. Monatsschr. 8 (1954), S. 190 f.; Franz Spechtler, Eine bisher unbekannte Handschrift des «Mönchs von Salzburg» in der Salzburger Studienbibliothek. Mitteilungen der Gesellschaft für Salzburger Landeskunde 102 (1962), S.35-49; Joachim Telle, Petrus Hispanus in der altdeutschen Medizinliteratur. Untersuchungen und Texte unter besonderer Berücksichtigung des «Thesaurus pauperum». Phil. Diss. Heidelberg 1972, S.92-97; Volker Zimmermann, Der Rosmarin als Heilpflanze und Wunderdroge. Sudhoffs Arch. 64 (1980), S.363-368.

4 Vgl. Gerhard Eis, Nachricht über eine altdeutsche Sammelhandschrift aus dem italienischen Kloster Farfa. Med. Monatsschr. 13 (1959), S.514-516; Volker Zimmermann, Rezeption und Rolle der Heilkunde (s. Anm.2), S. 14-16.

5 Davon tradiert das Salzburger Kompendium auf den Bll. $204^{\text {va }}, 205^{\mathrm{rb}}$, va, vb $209^{\mathrm{rb}}$, va $230^{\mathrm{va}, \mathrm{vb}}, 244^{\mathrm{ra}}, 248^{\mathrm{vb}}, 256^{\mathrm{ra}}$, rb $, 257^{\mathrm{va}}, 258^{\mathrm{rb}}, 395^{\mathrm{rb}}$, va, $396^{\mathrm{rb}}$ insgesamt 23 und der Kodex aus dem Benediktinerkloster Farfa in Latium auf den Bll. $98^{\mathrm{r}-\mathrm{v}}, 99^{\mathrm{r}-\mathrm{v}}, 122^{\mathrm{r}}, 125^{\mathrm{r}-\mathrm{v}}, 126^{\mathrm{v}}$, $181^{\mathrm{r}}$ insgesamt 15 entsprechende Rezepte zur Frakturbehandlung.

6 Vgl. A.G. Varron, Hygiene im Mittelalter. Ciba-Zeitschrift 74. Wehr/Baden (1955), S.2452-2457; Volker Zimmermann, Ansätze zu einer Sozial- und Arbeitsmedizin am mittelalterlichen Arbeitsplatz. In: Mensch und Umwelt im Mittelalter, hrsg. von Bernd Herrmann. Stuttgart 1986, S.140-149; derselbe, Krankheit und Gesellschaft: Die Pest. Sudhoffs Arch. 72 (1988), S. 6.

7 Beim «Bleiweiß» handelt es sich um basisches Bleikarbonat $2 \mathrm{PbCO}_{3} \cdot \mathrm{Pb}(\mathrm{OH})_{2}$. Vgl. Gundolf Keil, Die «Cirurgia» Peters von Ulm. Untersuchungen zu einem Denkmal altdeutscher Fachprosa mit kritischer Ausgabe des Textes. Phil. Diss. Heidelberg 1960 ( = Forschungen zur Geschichte der Stadt Ulm, Bd.2). Ulm 1961, S. 441; Dietlinde Goltz, Studien zur Geschichte der Mineralnamen in Pharmazie, Chemie und Medizin von den Anfängen bis Paracelsus (= Sudhoffs Arch. Beiheft 14). Wiesbaden 1972, S.246f.

8 Spangrün Synonym zu «Grünspan» ist basisches $\mathrm{Kupferazetat} \mathrm{Cu}\left(\mathrm{CH}_{3} \mathrm{COO}\right)_{2} \cdot \mathrm{Cu}(\mathrm{OH})_{2}$. Vgl. Gundolf Keil, Cirurgia (s. Anm. 7), S.465 f. und Dietlinde Goltz, Studien (Anm. 7), S. $135 \mathrm{f}$.

9 Vgl. Joachim Stürmer, Von deme Gîre. Untersuchungen zu einer altdeutschen Drogenmonographie des Hochmittelalters (=Würzburger medizinhistorische Forschungen, Bd. 12). Pattensen/Han. 1978, S.59.

10 Im mittelalterlichen «Buch von guten Pflastern und Salben» dient diese Drogenmasse als granulationsfördernder Bestandteil in einer Wundsalbe. Vgl. Dieter Lehmann, Zwei wundärztliche Rezeptbücher des 15.Jahrhunderts vom Oberrhein (=Würzburger medizinhistorische Forschungen, Bd.34). Pattensen/Han. 1985, S. 54 und 156.

11 Vgl. Heinrich Marzell, Geschichte und Volkskunde der deutschen Heilpflanzen. Darmstadt 1967, S.140-144; Hildegard von Bingen, Physica sive subtilitatum diversarum 
naturarum creaturarum Libri IX. In: J. P. Migne, Patrologiae cursus completus. Tom. CXCVII, Paris 1855, Sp. 1146 f.

12 Vgl. Heinrich Marzell, Heilpflanzen (s. Anm.11), S. 186 f.

13 Vgl. ebenda, S.239-245.

14 Vgl.ebenda, S.108-110; Hans-Dieter Stoffler, Der Hortulus des Walafrid Strabo. Sigmaringen 1978, S.96f.

15 Vgl. Otto Gessner, Gift- und Arzneipflanzen von Mitteleuropa, hrsg. und neu bearbeitet von Gerhard Orzechowski. 3. Auflage, Heidelberg 1974, S. 92 f, 165, 226-229, 411 f.

16 Vgl. C. Plinius Secundus, Naturalis Historiae Libri XXXVII, hrsg. von Carl Mayhoff. Leipzig 1897, Vol. IV, S. 242 (Lib. 27, 42).

17 Es handelt sich um die observationes 90 und 91 in der ersten centuria und um die observatio 90 in der dritten centuria. Vgl. Guilhelmus Fabricius Hildanus, Observationum et curationum chirurgicarum centuriae omnes. Lyon 1641, S. 116-118 und 505-507.

18 Vgl. Volker Zimmermann, Rezeption und Rolle der Heilkunde (s. Anm.2), S. 119.

19 Vgl. Guy de Chauliac, Chirurgia magna, hrsg. von Laurent Joubert. Lyon 1585. Neudruck mit einem Vorwort von Gundolf Keil. Darmstadt 1976, S.229.

20 Vgl. Arthur Hübner, Grundsätze für die Herausgabe und Anweisungen zur Druckeinrichtung der Deutschen Texte des Mittelalters. In: Johannes Rothe, Das Lob der Keuschheit, hrsg. von Hans Neumann (= Deutsche Texte des Mittelalters, hrsg. von der Preußischen Akademie der Wissenschaften, 38). Berlin 1934, S. V-IX.

\section{Summary}

Apart from those methods for the treatment of fractures that are given in the relevant chapters of the grand medieval works on surgery, the medicine of the Middle Ages was also familiar with specific procedures which were mainly based on indigenous remedies and which were primarily applied by lay-practitioners. Corresponding prescriptions which are passed and interspersed in compendious collective manuscripts are edited in context and examined here for the first time.

\section{Résumé}

Outre les méthodes chirurgicales concernant le traitement des fractures - décrites dans les grandes chirurgies du Moyen Age - la médecine médiévale en a connu d'autres qui se référaient primordialement aux plantes indigènes. Ces méthodes furent surtout pratiquées par des barbiers. C'est pour la première fois qu'on édite et analyse dans leur ensemble de telles leur prescriptions léguées d'une manière éparpillée dans des compendiums manuscrits.

Prof. Dr. phil. Dr. med. habil. Volker Zimmermann

Institut für Geschichte der Medizin der Universität Göttingen

Humboldtallee 11, D-3400 Göttingen 